\title{
Patients with anxiety and depression wanted to know what to expect when they started their medication
}

Haslam C, Brown S, Atkinson S, et al. Patients' experiences of medication for anxiety and depression: effects on working life. Fam Pract 2004;21:204-12.

\section{Q How do mental health problems and treatment of these problems affect working life?}

DESIGN

Qualitative study using focus groups.

\section{SETTING}

Leicestershire, UK.

\section{PATIENTS}

12 focus groups. 9 groups of patients who had anxiety and/or depression in the previous 2 years and used medication ( 6 groups: 35 patients [28-60 y, 63\% women] from various work sectors; 3 groups: 19 patients [ $18-63$ y, 74\% women] attending anxiety management courses). 3 groups of 20 staff who had responsibility for human resources, personnel, and occupational health and safety (age not reported, $65 \%$ women).

\section{METHODS}

Each focus group session (approximately $90 \mathrm{~min}$ ) was audiotaped and transcribed. Transcripts were analysed by sorting verbatim material into emergent themes using Knodel's method. An expert panel reviewed the findings to discuss implications for practice.

\section{MAIN FINDINGS}

Impact on working life. Participants shared how their families, friends, or colleagues often recognised their depression or anxiety symptoms first. Typically, participants were unaware until a crisis occurred. Symptoms included nausea, headache, dizziness, trembling, lack of energy, tiredness, lack of concentration, extremes of emotion, and lack of motivation. Sleep patterns were disturbed, which perpetuated the feeling of tiredness. Most respondents felt that their work performance was impaired: they were indecisive, unable to concentrate, and unproductive. Effects of prescribed medication. Drug side effects were similar to anxiety and depression symptoms (ie, confusion, dizziness, nausea, and difficulties with decision making). Participants described feeling distant and dislocated. Sometimes anxiety symptoms were exacerbated by the medication. Noncompliance with medication. Participants often took less than the prescribed amount or discontinued use altogether because their symptoms initially worsened or did not improve. Some experienced symptoms when they forgot to take their drugs or stopped treatment, which made them fear addiction or dependence. Participants who found the medication helpful worried about psychological dependency. Generally, participants did not like taking medication and would often stop once their symptoms began to improve. Patient information. Some participants found their GPs helpful. Many, however, found that they were not given enough information about side effects. The leaflets accompanying medications were frightening and unhelpful. Participants also had differing ideas about how long the medication needed to take effect and the importance of taking medication every day. Participants admitted to hospital found that hospital staff could take the time to explain fully what to expect from medication. Self help groups were also good sources of information. The expert panel felt that GPs needed to "sell" the benefits of medication and explain how patients might feel heightened anxiety initially. Patient monitoring. The expert panel felt that compliance was not as great a problem with more recent drugs. They also thought For correspondence: Professor $\mathrm{C}$ Haslam, Institute of Work, Health a Organisations, University of Nottingham, Nottingham, UK. Cheryl.Haslam@ nottingham.ac.uk

Source of funding: Health and Safety Executive. that lack of compliance was most problematic in the early stages of treatment, when patients may feel worse, and later on when they start to feel better. Most participants reported that their drug prescriptions were reviewed on a regular basis. Participants valued consistency in treatment. Sometimes they felt that GPs were not familiar with the medication or range of services. When referred to see a specialist, they felt that treatment options and possible causes of their illness were more fully discussed. Access to services varied among participants.

\section{CONCLUSIONS}

Patients with anxiety and depression felt that their work performance was impaired by their symptoms and drug side effects. They felt ill informed and needed more information to help them know what to expect so that they did not discontinue drugs prematurely.

\section{Commentary}

rhy he major strength of the study by Haslam et al is the identification of physical and psychological symptoms from medication side effects that affect work life and contribute to non-compliance by self identified patients with anxiety or depression. The findings are consistent with the interaction problems between patients and physicians that were identified in a previous study by Young et al. ${ }^{1}$ The current study found that patients' lack of education about their illnesses and medication side effects contributed to non-adherence with medication regimens. Miscommunication and lack of understanding between patients and their care providers show the importance of the relation between interactions and adherence. Rigour was ensured by having 3 research team members systematically review the qualitative findings to determine the reliability of the resulting themes.

The themes that emerged applied to a broad category of anxiety and mood disorders rather than a single diagnosis. The authors do not differentiate between themes found in workers and manager/employer groups, and reported themes seem to apply to workers' experiences. No illustrative quotes were used from the employer groups. The study highlights several problems affecting workers coping with mental illness and its treatment and the need for improvement in the recognition and provision of psychiatric treatment. Surprisingly, others in the workplace recognised participants' symptoms before participants did.

The findings suggest that nurses and the healthcare team in general practice and the community need to provide more effective psychoeducation to patients. Increased patient knowledge of disorders, including prognosis, medication treatment, potential side effects, and need for treatment continuation and monitoring, are important factors in assuring patient adherence. Study patients reported that education provided by specialists in hospital was superior to that provided by general practitioners in the community, which suggests a need for greater and more timely collaboration among providers. The findings also suggest the need for a system of care that systematically implements comprehensive psychoeducation and follow up.

New strategies for the management of anxiety and mood disorders as experienced in the workplace need to be investigated and tested. Differentiating employers' perceptions from workers' perceptions may yield important findings in future studies, and gender differences should also be highlighted and explained.

Sarah Mynatt, RN, EdD, APN University of Tennessee Health Sciences Center Memphis, Tennessee, USA

1 Young AS, Klap R, Sherbourne CD, et al. The quality of care for depressive and anxiety disorders in the United States. Arch Gen Psychiatry 2001;58:55-61. 